\title{
Metastatic Microcystic Adnexal Carcinoma with DNA Sequencing Results and Response to Systemic Antineoplastic Chemotherapy
}

\author{
MIN-BIN CHEN ${ }^{1}$ and DAMIAN A. LABER ${ }^{2}$ \\ ${ }^{1}$ Department of Oncology, Kunshan First People's Hospital Affiliated to Jiangsu University, Kunshan, P.R. China; \\ ${ }^{2}$ Section of Satellite Oncology, Moffitt Cancer Center and Division of Hematology/Oncology, \\ Morsani School of Medicine, University of South Florida, Tampa, FL, U.S.A.
}

\begin{abstract}
Microcystic adnexal carcinoma $(M A C)$ is a rare cutaneous malignancy. Due to its rarity, the molecular characteristics and treatment for metastatic MAC remain undefined. Here we present, as far as we are aware, the first case of metastatic MAC with DNA sequencing results indicating a mutation in TP53 and chromosomal losses in cyclin dependent kinase inhibitor $2 A(C D K N 2 A)$ and cyclin dependent kinase inhibitor $2 B(C D K N 2 B)$. In addition, this is the first case of metastatic MAC with a documented objective response to systemic antineoplastic chemotherapy (carboplatin and paclitaxel) confirmed by positron emission tomography/computed tomography. Our case increases the very limited medical knowledge of this rare disease.
\end{abstract}

Microcystic adnexal carcinoma (MAC) is a rare cutaneous tumor which was first described as a separate clinical entity by Goldstein et al. in 1982 (1). All medical knowledge comes from case reports and small retrospective studies $(2,3)$. Only four cases of systemic metastasis have been reported in the literature and none received systemic therapy (4-7). Here we describe a man with a metastatic MAC, including the results of DNA sequencing of the tumor. He received antineoplastic chemotherapy and achieved an excellent response. To our knowledge, this is the first report in the medical literature of such findings.

Correspondence to: Damian A. Laber, MD, FACP, 3 Tampa General Circle, Tampa, FL 33606, U.S.A. Tel: +1 8139743725, Fax +1 8139749476, e-mail: damian.laber@gmail.com

Key Words: Microcystic adnexal carcinoma, carboplatin, paclitaxel, next-generation sequencing.

\section{Case Report}

A 68-year-old man developed a subcutaneous nodule in the superior aspect of his left thumb. Excisional biopsy revealed microcystic adnexal adenocarcinoma and he received radiotherapy. Three years later, he developed edema of the left arm, forearm and hand. Computed tomographic scan (CT) showed axillary lymphadenopathy. Biopsy of his left axillary lymph node (LN) revealed microcystic adnexal adenocarcinoma. His physical examination revealed pitting edema over his left arm, forearm and hand; a palpable left supraclavicular LN approximately $3 \mathrm{~cm}$ in size and a few LNs less than $1 \mathrm{~cm}$ palpable in the left axilla. The rest of the physical examination was normal. Positron-emission (PET)/CT) showed a single left supraclavicular LN with a standardized uptake value (SUV) of 5.8, multiple left axillary LNs with SUV of 3.5-6.9, single foci of increased activity in the liver with SUV of 8.1, a single foci of increase activity in the spleen with SUV of 7.1 and pulmonary nodules less than $1 \mathrm{~cm}$. There were no other abnormalities. Brain magnetic resonance imaging showed no lesions.

The pathology was reviewed by two separate academic institutions and both confirmed the diagnosis. The morphology showed the presence of an adenocarcinoma with a small component of sarcomatous differentiation. Immunohistochemical staining revealed malignant epithelial nests staining positively for pankeratin, cytokeratin $5 / 6$ (CK5/6), anti-cytokeratin (CAM 5.2), epithelial cell adhesion molecule/epithelial specific antigen (MOC31) and luminal positivity for carcinoembryonic antigen (CEA). The spindled stromal cells were positive for smooth muscle actin and negative for desmin and S- 100 .

Next-generation sequencing was performed on DNA extracted from tumor tissue with a panel of 435 genes for solid tumors, including potential response to immune checkpoint inhibitors (8). The tumor harbored a mutation in 
TP53 and chromosomal losses in cyclin dependent kinase inhibitor 2A $(C D K N 2 A)$ and cyclin dependent kinase inhibitor $2 \mathrm{~A}(C D K N 2 B)$. It had no mutations in epidermal growth factor receptor (EGFR), kirsten Ras (KRAS), neuroblastoma Ras $(N R A S)$, V-Raf murine sarcoma viral oncogene homolog B (BRAF), mesenchymal-epithelial transition factor $(M E T)$, or human epidermal growth factor receptor 2 (HER2); no rearrangements in anaplastic lymphoma kinase $(A L K)$, rearranged during transfection (RET), or proto-oncogene 1 receptor tyrosine kinase (ROS1); and a non-hypermutated phenotype.

After obtaining informed consent, the patient received therapy with paclitaxel $\left(175 \mathrm{mg} / \mathrm{m}^{2}\right)$ and carboplatin [area under the curve (AUC) 5] every 3 weeks for four cycles with no significant side-effects. One month after completing chemotherapy, his LNs could no longer be palpated. PET/CT showed resolution of the previously noted metabolic activity in the LNs, lung, liver and spleen and reduction in the size of all lesions consistent with an objective partial response. Three months later, he remained asymptomatic. Surveillance CT scans showed some pulmonary nodules to be smaller, some stable and some minimally larger in size. It also revealed development of sclerotic spine lesions, reduction in size of previous hepatic and splenic metastases, but multiple additional liver lesions. CT scans 2 months later revealed progressive disease everywhere. At the time of preparation of this article, the patient had been scheduled to restart chemotherapy with carboplatin and paclitaxel while requesting support for therapy with Inhibitors of CDK4 and -6 .

\section{Discussion}

Here, as far as we are aware, we report for the first time in medical literature the detailed molecular profile and response to systemic antineoplastic chemotherapy of a patient with metastatic MAC. Our patient initially was treated with surgical resection and adjuvant radiotherapy. He developed symptomatic metastases approximately 3 years later. The sites of metastases included the axillary and supraclavicular LNs, lungs, liver, spleen and bones. Distant metastases have been described in the medical literature in only four cases of MAC (4-7). The previously described metastatic sites included skin, LNs, bone, liver and lung (4-7). A review of the National Cancer Institute Surveillance, Epidemiology, and End Results (SEER) database from 1973 to 2004 found 223 cases of MAC, only one reported distant metastases (2). The overall survival was similar to the standard population matched for the same years, suggesting the low metastatic potential of this malignancy (2).

Our patient received four cycles of carboplatin and paclitaxel. He achieved a partial response confirmed by CT with normalization of all PET glucose-avid areas and complete resolution of his cancer-related symptoms which lasted for 3-5 months. We could not find any documented cases of the use of systemic therapy for metastatic disease in the medical literature. A single course of cisplatin and 5fluorouracil chemotherapy for locally recurrent MAC was reported once in 1995, which was deemed non-effective and the patient was treated with surgical resection and radiotherapy (9). The review of the SEER database from 1973 to 2004 found no documentation of the use of systemic chemotherapy (2).

We sequenced extended coding regions of over 435 genes using next-generation sequencing technology (8). We found two actionable mutations $C D K N 2 A$ loss and $C D K N 2 B$ loss. Inhibitors of CDK4 and - 6 have been developed and are under investigation for cancer expressing these molecular markers, with one recently approved by the Food and Drug Administration for subsets of patients with breast cancer (10). These agents might be a potential treatment option for our patient.

In conclusion, our case represents several firsts regarding reports of MAC in the medical literature: the first report of metastasis to the spleen, first report of systemic antineoplastic chemotherapy for metastatic disease, first report of response to systemic chemotherapy, and first report of the use of next-generation sequencing in this disease. Due to the extreme rarity of this disease, all these finding are very valuable in our understanding of MAC. We hope that our case will stimulate other publications and increase the medical knowledge of this rare disease.

\section{Conflicts of Interest}

The Authors received no funding for this work and have no conflicts of interest related to this work or financial relationships to report.

\section{References}

1 Goldstein DJ, Barr RJ and Santa Cruz DJ: Microcystic adnexal carcinoma: a distinct clinicopathologic entity. Cancer 50: 566$572,1982$.

2 Yu JB, Blitzblau RC, Patel SC, Decker RH and Wilson LD: Surveillance, Epidemiology, and End Results (SEER) database analysis of microcystic adnexal carcinoma (sclerosing sweat duct carcinoma) of the skin. Am J Clin Oncol 33: 125-127, 2010.

3 Hansen T, Kingsley M, Mallatt BD and Krishnan R: Extrafacial microcystic adnexal carcinoma: case report and review of the literature. Dermatol Surg 35: 1835-1839, 2009.

4 Carroll P, Goldstein GD and Brown CW Jr.: Metastatic microcystic adnexal carcinoma in an immunocompromised patient. Dermatol Surg 26: 531-534, 2000.

5 Ohta M, Hiramoto M and Ohtsuka H: Metastatic microcystic adnexal carcinoma: an autopsy case. Dermatol Surg 30: 957-960, 2004.

6 Chaudhari S, Blanchard L, Chaudhari PJ and Lythgoe T: The first reported case of metastatic microcystic adnexal carcinoma in an adolescent. Dermatol Surg 41: 286-288, 2015. 
7 Gabillot-Carre M, Weill F, Mamelle G, Kolb F, Boitier F, Petrow P, Ortoli JC, Margulis A, Souteyrand P, Mercier S, Spatz A, Duvillard P, Validire P and Avril MF: Microcystic adnexal carcinoma: report of seven cases including one with lung metastasis. Dermatology 212: 221-228, 2006.

8 Weinstein JN, Collisson EA, Mills GB, Shaw KR, Ozenberger BA, Ellrott K, Shmulevich I, Sander C and Stuart JM: The Cancer Genome Atlas Pan-Cancer analysis project. Nat Genet 45: 1113-1120, 2013
9 Bier-Laning CM, Hom DB, Gapany M, Manivel JC and Duvall AJ 3rd: Microcystic adnexal carcinoma: management options based on long-term follow-up. Laryngoscope 105: 1197-1201, 1995.

10 Sherr CJ, Beach D and Shapiro GI: Targeting CDK4 and CDK6: From Discovery to Therapy. Cancer Discov 6: 353-367, 2016.

Received June 23, 2017

Revised July 17, 2017

Accepted July 18, 2017 